\title{
Synchrotron Radiation Production and Trapping of Highly Charged Ions
}

\author{
B. M. Johnson, K. W. Jones and M. Meron \\ Brookhaven National Laboratory, Upton, New York 11973
}

\author{
Invited Talk, Presented at \\ Eighth Conference on the \\ Application of Accelerators in Research and Industry \\ Denton, Texas \\ November $12-14,1984$
}

\section{DISCLAIMER}

\begin{abstract}
This report was prepared as an account of work sponsored by an agency of the United States Government. Neither the United States Government nor any agency thereof, nor any of their employees, makes any warranty, express or implied, or assumes any legal liability or responsibility for the accuracy, completeness, or usefulness of any information, apparatus, product, or process disclosed, or represents that its use would not infrings privately owned rights. Reference herein to any specific commercial product, process, or service by trade name, trademark, manufacturer, or otherwise does not necessarily constitute or imply its endorsement, recommendation, or favoring by the United States Government or any agency thereof. The views and opinions of authors expressed herein do not necessarily state or reflect those of the United States Government or any agency thereof.
\end{abstract}

The submitted manuscript has been authored under contract DE-AC02-76CH00016 with the Division of Basic Energy Sciences, U. S. Department of Energy. Accordingly, the U. S. Government retains a nonexclusive, royalty-free license to publish or reproduce the published form of this contribution, or allow others to do so, for U. S. Government purposes. 
SYNCHROTRON RADIATION PRODUCTION AND TRAPPING OF HIGHLY CHARGED IONS*

B. M. Johnson, R. W. Jones and M. Meron

Brookhaven National Laboratory, Upton, New York 11973

\begin{abstract}
Wiggler-enhanced syrichrotron radiation will soon be available at dedicated facilities such as the National Synchrotron Light Source (NSLS) at Brookhaven National Laboratory. Two recent proposals to study highly-charged ions produced by successive photoionization with broad-band synchrotron radiation are based on the design parameters of the NSLS $x$-ray ring. Previous calculations indicated that in a suitable Kingdor. trap, concentrations of $\mathrm{S} \times 10^{7} \mathrm{Ar}^{18+}$ j.ons per $\mathrm{Cm}$ can be attained in a few seconds trapping time and that in a Penning trap $5 \times 10^{3} \mathrm{Ar}^{17}+$ ions could be stored at near thermal energies $\left(300^{\circ} \mathrm{K}\right)$ to study, for example, charge transfer collision processes for cold, highly-charged ions. Here the previous work is reviewed and the earlier calculations are extended. The addition of an axial magnetic field to a Kingdon trap is shown to improve expected PHOBIS performance.
\end{abstract}

*Research supported by the Fundamental Interactions Branch, Division of Chemical Sciences, Office of Basic Energy Sciences, US Department of Energy, Contract No. DE-AC02-76CH00016. 


\section{Introduction}

The sucessive photoionization of trapped ions has been proposed recently $[1,2]$ as a viable new method for producing, storing, and observing highly-charged lons. The basic idea of PHOBIS, the PHOton Beam Ion Source, is to confine ions il. a potential well and produce large concentrations of highly-charged lons through multi-step photoionization by wiggler-enhanced synchrotron radiation. The fons can then be efther extracted for external experiments or studied in situ. There is much interest in this concept, because versatile ion sources for the production and study of any desired element, charge state, and beam energy are needed to extend and improve basic atomic physics experiments and to meet the atomic data needs of high-temperature p] esma research.

In general, high-charge-state ions can be produced in one of two ways: by acceleration to high ion energy with electron strippiug by passage through foil or gas and by confinement at low energy with electron stripping by bombardment. The acceleration approach can provide high beam currents and high charge states at beam energies of tens to hundreds of $\mathrm{MeV}$ or, with the recently developed accel-decel method [3], at energies as low as a few MeV. Production at lower ion beam energies requires bombardment sources [4] such as the electron beam ion source (EBIS), the electron cyclotron resonance ion source (ECRIS), and the recoil ion source. For these sources, ion beam currents are generally lower than for beams from accelerators, especially for very high charge states. Furthermore, the presence of 
extraneous charged particles complicatea source design thile precluding the abllity to use spectroscopy of the trapped fons as a diagnostic tool. For bombardment by intense broad-band synchrotron radiation, however, the uncharged lonizing agent simplifies trapping considerations while affording the opportunity to study charge exchange and other ion-ion interactions in a clean environment at very low collision energies.

The basic PHOBIS concept is a logical, perhaps even obvious, extension of the principles of charged-particle bombardment sources. What is not obvious is the idea that successive photoionization of trapped fons will yield sufficient numbers of highly-charged ions to make a reasonable ion source. Nevertheless, the calculations indicated not only that such an approach is feasible with the very-high-flux white $x$-ray beams avallable from dedicated high-performance synchrotron storage rings, but also that very-highly ionized lons can be produced independent of a particular cholce of trap, and moreover that the performance Jevels with a Kingdon trap $[5,6]$ can be comparable to existing sources. In a subsequent paper [2] the calculations were extended to another application of the PHOBIS concept, the production and storage in a Penning trap [7] of multi-charged lons with mean energies near room temperature. In the present paper, the previous discussions $[1,2]$ are reviewed, improvements in trap design are considered, and the initial experiments planned for the dedicated atomic physics beam line at the National Synchrotron Light Source (NSLS) are described. 


\section{PHOBIS, the FHOton Bean Ion Source}

Independent of a particular cholce of trap, the PHOBIS Ion source is shown schemacically in fig. 1. To obtain quantitative estimates of PHOBIS performance, it is assumed [1] that a 10-mr sagittal portion of the photon beam is focussed by a mirror tnto a spot which is $0.2 \mathrm{~cm}$ high by $1.0 \mathrm{~cm}$ long or an area of $0.2 \mathrm{~cm}^{2}$. The photon beam intensity and energy distribution are taken from the design parameters [8] for the NSLS x-ray ring operating at $2.5 \mathrm{GeV}$ with 500-mot electron beam current and a 25-pole wiggler to increase ilux and photon energy range. Inftial estimates of lonfzation times were first obtalned [1] using a simple independent-electron model (IEM). This model assumes successive single ionization to be the dominant ionization mechanism for the trapped fons and ignores roughly offsetting contributions from multiple ionization $[9,10]$ and successively increasing binding energles.

As shown in [1], convoluting the photon flux distribution with the energy dependent photoionization cross section yields the photolonization rate,

$$
\tau_{1}^{-1}=\int_{0}^{\infty} N_{x}(E) \sigma(E) d E
$$

at energy $E$, and $\sigma(E)$ is the photolonization cross section per electron in shell 1 . After the onset of ionization, the number of electrons remaining in the shell $i$ at time $t$ is given by

$$
N_{e}(t) / N_{e}(0)=e^{-t / \tau_{i}}
$$


and the average time for full ionization is given by

$$
T_{1}=\tau_{1} \sum_{k=1}^{N_{1}} 1 / k
$$

where $\mathbb{N}_{1}$ is the initial number of electrons in the shell.

Table 1 gives the total ionization times $T_{1}$ obtained for the argon $M, L$, and $K$ shells from the uncorrected IEM calculation (A), for corrections to IEM for the effects of multiple ionization and the filling of inner-she1l vacanzies by outer electrons (B), and for full correrions including these effects plus the charge-state depentence of binding energies (C). Note in Table 1 that the B calculation gives shorter times for the $M$ shell where multiple ionization effegts are largest, and somewhat longer times for the $L$ and $K$ shells, whereas the C calculation gives somewhat longer lonization times than A for all shells. These calcualations indicate that irradiation of a given volume of an unspecified trap for a few seçonds will produce high charge states up to and including completely stripped Ar ions.

\section{The Kingdon Trap}

Fig. 2 shows a schematic representation of a Kingdon trap $[5,6]$, which is considered [1] to be the best PHOBIS candidate for efficient ion extraction to produce an external beam. Basically, the trap is two concentric cylinders with inner and outer radil $a$ and $b(a<<b)$ with a potential difference $V$ between them and two end caps at tine same electrostatic potential as b. The only trapping field is electrostatic and the trapping potential is logarithmic. 
In CGS units the number of trapped Ions per unit length $\lambda$ which can be kept in the trap is given by

$$
\lambda=v / 2 \ln (b / a) / \text { eq }
$$

where $q$ is the charge state of the ion of interest.

Construction of such a trap is subject to several practical constraints. For example, the applied voltage is limited to the breakdown voltage $V_{c}$ given by

$$
v_{c}=\varepsilon_{c} a \ln (b / a)
$$

where $\varepsilon_{c}$ of order $3 \times 10^{4} \mathrm{~V} / \mathrm{cm}$ is the maximum local field before breakdown occurs. This and relation (4) seem to favor large values of a. However, as the lons are created with a very low kinetic energy, their orbits are highly eccentric with "periaxis" much smaller than "apoaxis". Assuming that the ions are created near the outer wall of the trap, with a random initial distribution of velocitles, and that collisions with the central wire are to be avolded, $a$ and $b$ must fulfill the inequality

$$
(\mathrm{b} / \mathrm{a})^{2}>3 \mathrm{Veq} / \mathrm{K}
$$

where $\mathrm{K} / 3$ is the part of the average initlal kinetic energy related to transverse motion. As $K$ is usually not much larger than thermal energles, b/a should be large, on the order of $10^{2}-10^{3}$.

Table 2 gives the typlcal operating parameters for a reasonably sized PHOBIS trap. As Indicated in Fig. 2, ions could be extracted. efther radially or axially. Assuming efficient ion extraction, external Ion beams can be produced with intensities determined chiefly by the ability of the trap to confine large numbers of highly charged 
ions, i.e. large $\lambda$. The maximal value of $\lambda$ is determined by the breakdown voltage $V_{c}$ as given in eqn. (5). If a way can be found to avoid collisions with a thicker central wire (larger a), $V_{C}$ can be increased.

One approach developed here, to improve the performance of a Kingdon trap, is to apply an axial magnetic field. This B field will reduce the eccentricity of the orbits of the trapped ions and the Inequality for $a$ and $b$ given in relation (6) becomes

$$
(b / a)^{2}>(3 \operatorname{Veq} / K) /\left(1+3 b^{2} e^{2} q^{2} B^{2} / 8 K M c^{2}\right)
$$

in CGS units, where $M$ is the mass of the ion.

Table 3 gives optimum values of $\lambda$ for fixed outer radius $b$ as a function of applied axial magnetic field B. First relation (7) is treated as an equality, then $V$ is set equal to $\bar{v}_{c}$ from equation (5). For a given $B$, this results in two equations, (4) and (7), in two unknowns, $a$ and $\lambda$.

Fig. 3 shows the variation of $V$ and $\lambda$ versus $B$ for the same geometry as Table 2 (dashed curves), and for a four times larger inner radius (solid curves). As befure for given B relation ( 7 ) is taken as an equality. For fixed $a$ and $b, V$ is then given directly by equation (7), with the constraint that $V$ cannot be greater than $V_{c}$. Substitution of $V$ in equation (4) gives $\lambda$. Note that a thicker central wire results in degraded operation for $B=0$, but much improved performance at high $B$. 


\section{The Penning Ion Trap}

A Penning trap is the best choice for the study of ion-ion interactions within a trap. The advantage here would be to eliminate both the energy increment added by the extraction process, and the problem of avolding collistons with the central wire of a Kingdon trap. Ion-ion collision measurements could then be extended down to near-thermal energies. Although a Kingdon trap has been used for measurements such as electron capture of trapped $\mathrm{Ne}^{q^{+}}$ions at mean collision energies from $1.0-70.0 \mathrm{eV}$ [10], it was shown [2] that a Penning trap [6] could provide the opportunity to study trapped $\mathrm{Ar}^{12+}$ Ions at mean energies near $0.025 \mathrm{eV}=300 \mathrm{~K}$, that is at room temeprature.

Fig. 5 shows a schematic representation of a Penning trap [7], which consists of a cylindrically symmetrical electrostatic quadrupole potential and a superimposed axial magnetic field. (Note the absence of a central wire.) The figure also shows the radio fraquency excitation circuits for detection and charge-to-mass ratio selection of stored ions, based on cyclotron excltation.

A detalled discussion of the application of Penning traps will not be repeated here, but Table 4 gives typical operating parameters [2] for a suitable PHOBIS trap. For further information, the reader is referred to [2] and [6] and references therein.

\section{Sumary and Conclusions}

A new ion source for the production and storage of highly-charged Ions, PHOBIS - the Photon Beam Ion Source, has been reviewed and 
previous calculations of expected performance have been extended. The design parameters for wiggler-enhanced synchrotron radiation from the NSLS x-ray ring were used to predict expected operating parameters of PHOBIS for $\mathrm{Ar}^{+}$with $\mathrm{q}$ as high as 18 (fully-stripped argon). After the general perfortance with an unspecified trap was considered, two particular ion traps were chosen to be best suited for the two alternative approaches to PHOBIS: extraction of lons to produce an external beam and observation of the interaction of fons within the trap.

An Ion source is generally considered to be a device that produces a beam of 1ons. A Kingdon trap was chosen for this application of PHOBIS, because the design and construction are simple and efther radial or axial fon extraction is expected to be efficient. Typical operating parameters for a conventional kingdon trap were summarized, and then the addition of an axial magnetic field was shown to improve expected performance.

The alternative PHOBIS approach is to study atomic collisions wthin the ion trap. The Idea here is to extend measuremerits to extremely low collision energies. Since any very low energy ion in a Kingdon trap will be lost by collision with the central wire, a Penning trap with no central wire was chosen for this approach to PHOBIS. - A summary of calculated performance for typical opertation Indicated that $\mathrm{Ar}^{\mathrm{I} 2+}$ ions could be trapped at room temperature, $300 \mathrm{~K}$ or $25 \mathrm{meV}$. 
The conclusion is that PHOBIS, an Ion source based on photolonization of trapped ions, is feasible, cheap, and simple to construct and operate. Design and construction of sultable traps is well underway and inftial experiments are planned for early 1985.

The development of a Penning trap for PHOBIS is a joint project with Professors D.A. Church and I.A. Sellin. We are Indebted to Dr. M.H. Prior for helpful discussions on the use of the Kingdon trap. 


\section{REFERENCES}

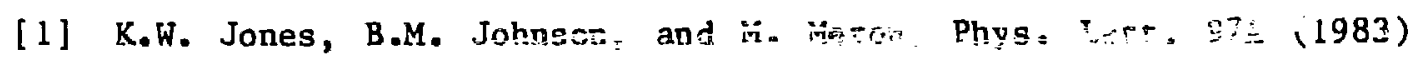
377.

[2] D.A. Church, K.W. Jones, B.M. Johnson, M. Meron, and I.A. Sellin, J. Phys. B: At. Mol. Phys. 17 (1984) L401.

[3] P. Thteberger, J. Barrette, B.M. Johnson, K.W. Jones, M. Meron, and H.E. Wegner, IEEE Trans. on Nucl. Sci. NS-30 (1983) 1431.

[4] V.0. Kostroun, "Proceedings of X-Ray and Atomic Inner-Shell Physics - 1982," AIP Conference Proceedings, No. 94, ed. by Bernd Crasemann, New York (1982) 303-317.

[5] R.H. Hooverman, J. App 1.. Phys. 34 (1963) 3505.

[6] M.H. Prior, R. Marrus, and C.R. Vane, Phys. Rev. A28 (1983) it1; and C.R. Vane, M.H. Prior, and R. Marrus, Phys. Rev. Lett. 46 (1981) 107 .

[7] J.B. Byrne and P.S. Farago, Proc. Phys. Soc. 88 (1965) 801.

[8] A. Van Steenbergen and INSLS Staff, Nuc1. Instrum. and Meth. 172 (1980) 25-32.

[9] T.A. Carlson, Phys. Rev. 151 (1966) 41; J.B. Hastings and V.0. Kostroun, BNL 31970, and Nucl. Instrum. Meth., to be published. 


\section{Table 1}

Average times in seconds for full iontzation of each shell of Ar in a PHOBIS source from simplea) (A), corrected $\left.{ }^{b}\right)$ (B), and fully correctedc) (C) calculations.

\begin{tabular}{cccc}
\hline Calculation & $\mathrm{T}_{\mathrm{M}}(\mathrm{s})$ & $\mathrm{T}_{\mathrm{L}}(\mathrm{s})$ & $\mathrm{T}_{\mathrm{K}}(\mathrm{s})$ \\
\hline $\mathrm{A}^{\mathrm{a})}$ & 0.09 & 0.53 & 2.7 \\
$\mathrm{~B}^{\mathrm{b})}$ & 0.06 & 0.57 & 3.2 \\
$\mathrm{C}$ & 0.13 & 1.9 & 6.9 \\
\hline
\end{tabular}
a) Independent electron model (IEM) assuming only successive single ionization.
b) Corrections to IEM. for effects of multiple ionization and filling of inner-shell vacancies.
c) Corrections to IEM for effects of multiple ionization, filling of inner-shell vacancies, and the charge-state dependence of binding energies.




\section{Table 2}

Typical parameters for a Kingdon trap for PHOBIS. Performance characteristic for $\mathrm{Ar}^{18+}$.

\begin{tabular}{|c|c|c|}
\hline Parameter & Symbol & Typical value \\
\hline Inner radius & $\mathbf{a}$ & $0.01 \mathrm{~cm}$ \\
\hline Outer radius & $\mathrm{b}$ & $4 \mathrm{~cm}$ \\
\hline Operating pressure & $\mathbf{P}$ & $1 \times 10^{-8}$ Torr \\
\hline Applied voltage & $\mathrm{V}$ & $300 \mathrm{~V}$ \\
\hline Trapped ions $\left(\mathrm{Ar}^{18+}\right)$ & $\mathrm{N}_{\mathrm{T}}$ & $1 \times 10^{7}$ Ions $/ \mathrm{cm}$ \\
\hline
\end{tabular}


Table 3

Optimum values of $\lambda$ in a modified Kingdon trap for PHOBIS, with fixed outer radius $b$ as $a$ function of applied axlal magnetic field $B$. Calculations are for Ax ${ }^{18+}$ lons according to relations $(4),(5)$ and (7) assuming an outer radius $b$ of $4.0 \mathrm{~cm}$, a breakdown fleld $\varepsilon_{c}$ of 3 $x 10^{4} \mathrm{~V} / \mathrm{cm}$, and an initital ton energy $\mathrm{K}$ of $0.1 \mathrm{eV}$.
B (gauss)
a (cm)
V (volts)
$\lambda(1 \mathrm{on} / \mathrm{cm})$.

\begin{tabular}{llll}
\hline 0 & $5.30 \times 10^{-3}$ & $1.05 \times 10^{3}$ & $3.07 \times 10^{7}$ \\
10 & $9.75 \times 10^{-3}$ & $1.76 \times 10^{3}$ & $5.64 \times 10^{7}$ \\
20 & $1.51 \times 10^{-2}$ & $2.53 \times 10^{3}$ & $8.76 \times 10^{7}$ \\
30 & $2.00 \times 10^{-2}$ & $3.18 \times 10^{3}$ & $1.16 \times 10^{8}$ \\
40 & $2.45 \times 10^{-2}$ & $3.74 \times 10^{3}$ & $1.41 \times 10^{8}$ \\
50 & $2.86 \times 10^{-2}$ & $4.24 \times 10^{3}$ & $1.66 \times 10^{8}$ \\
60 & $3.26 \times 10^{-2}$ & $4.70 \times 10^{3}$ & $1.88 \times 10^{8}$ \\
70 & $3.64 \times 10^{-2}$ & $5.13 \times 10^{3}$ & $2.10 \times 10^{8}$ \\
80 & $4.00 \times 10^{-2}$ & $5.53 \times 10^{3}$ & $2.31 \times 10^{8}$ \\
90 & $4.35 \times 10^{-2}$ & $5.90 \times 10^{3}$ & $2.52 \times 10^{8}$ \\
100 & $4.70 \times 10^{-2}$ & $6.26 \times 10^{3}$ & $2.72 \times 10^{8}$ \\
\hline
\end{tabular}




\section{Table 4}

Typical parameters [2] for a Penning trap for PHOBIS.

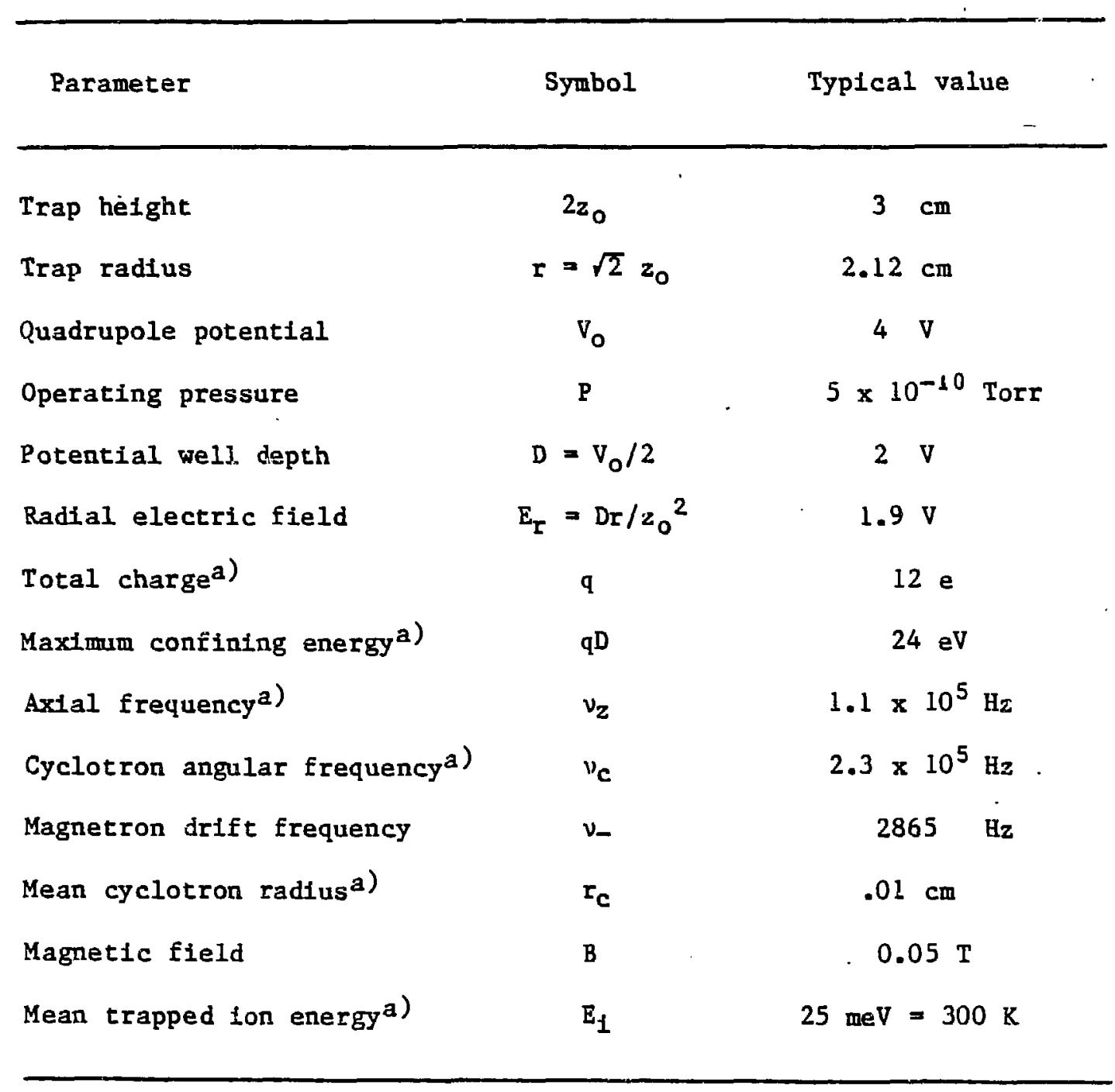

a) Performance characteristics for Ar ${ }^{12+}$. 


$$
-16-
$$

\section{FIGURE CAPTIONS}

Fig. 1. Conceptual design of PHOBIj apparatus. Many possible types of traps and experimental geometries can be envisaged for different experiments. Two possibilities are indicated: the extraction of ions from the trap and the study of radiation from interactions within the trap.

Fig. 2. Schematic representation of a Kingdon trap for PHOBIS. A logarithmic trapping potential is produced by a voltage $V$ between the central wire or inner cylinder $A$ (radius $a$ ) and the outer cylinder B (radius b). Sultable openings in the endcaps allow synchrotron radiation (photons) to pass through the trap. Ions could be extracted either radially or axially. See Table 2 and text for geometrical and operating parameters.

F1g. 3. Plots of voltage (a) and trapped charge per unit length (b) for a modified Kingdon trap as a function of applied magnetic field for two fixed geometries: $a=.01 \mathrm{~cm}$, $\mathrm{b}=4 \mathrm{~cm}$ (dashed curves) and $\mathrm{a}=.03 \mathrm{~cm}, \mathrm{~b}=4 \mathrm{~cm}$ (solid curves).

Fig. 4. Schematic representation of a Penning trap for PHOBIS. The cross-sectional view (upper) shows the introduction of synchrotron radiation (photons) into the trap, which is axially symmetric about the vector indicating the direction of the magnetic field, $B$. The representation of trap-ring electrodes (lower) Indicates the slots for introduction of synchrotron raditation and the radio frequency excitation circults for detection and charge-to-mass ratio of stored fons. 


\section{PHOBIS: PHOton Beam lon Source}

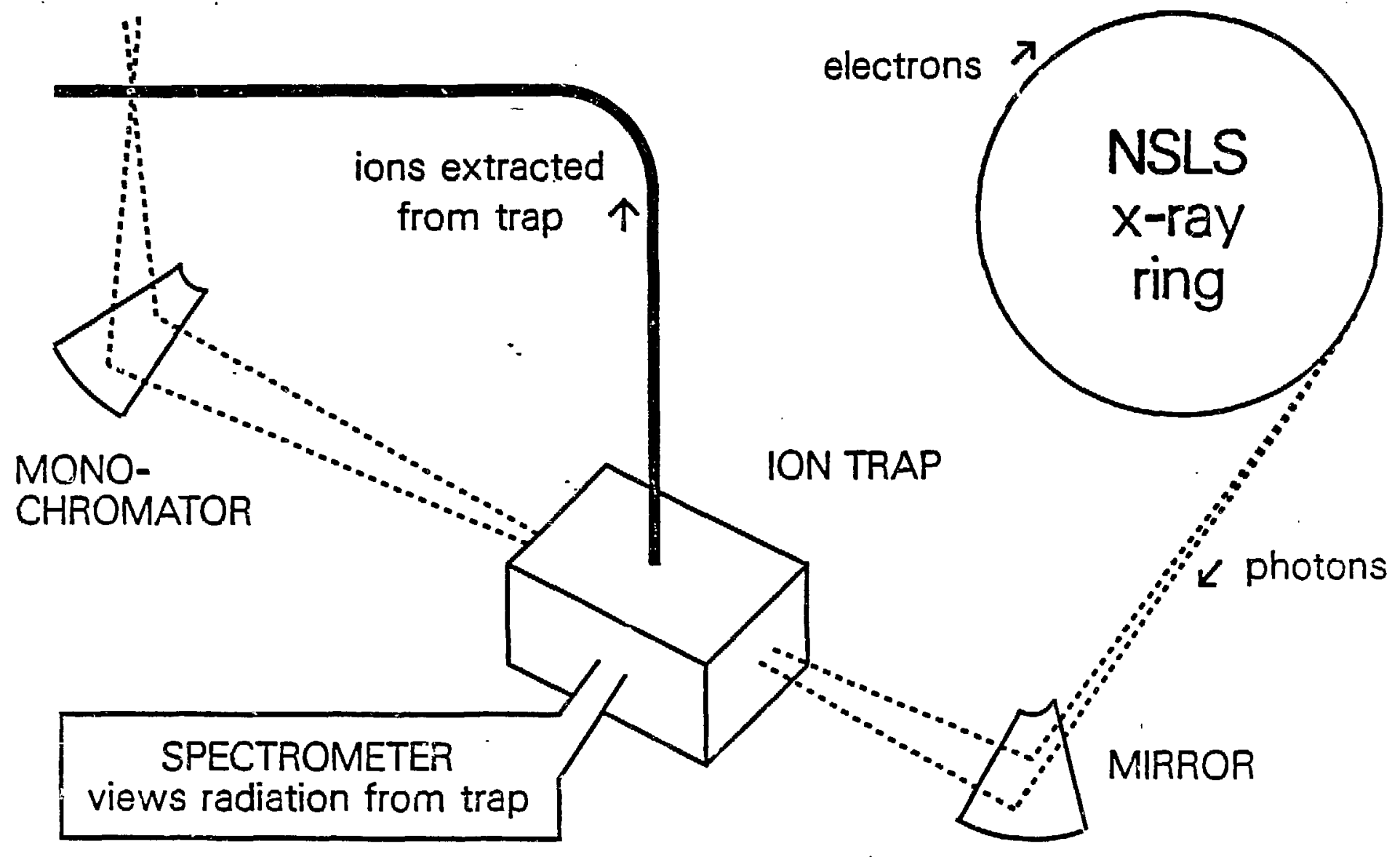




\section{KINGDON ION TRAP}
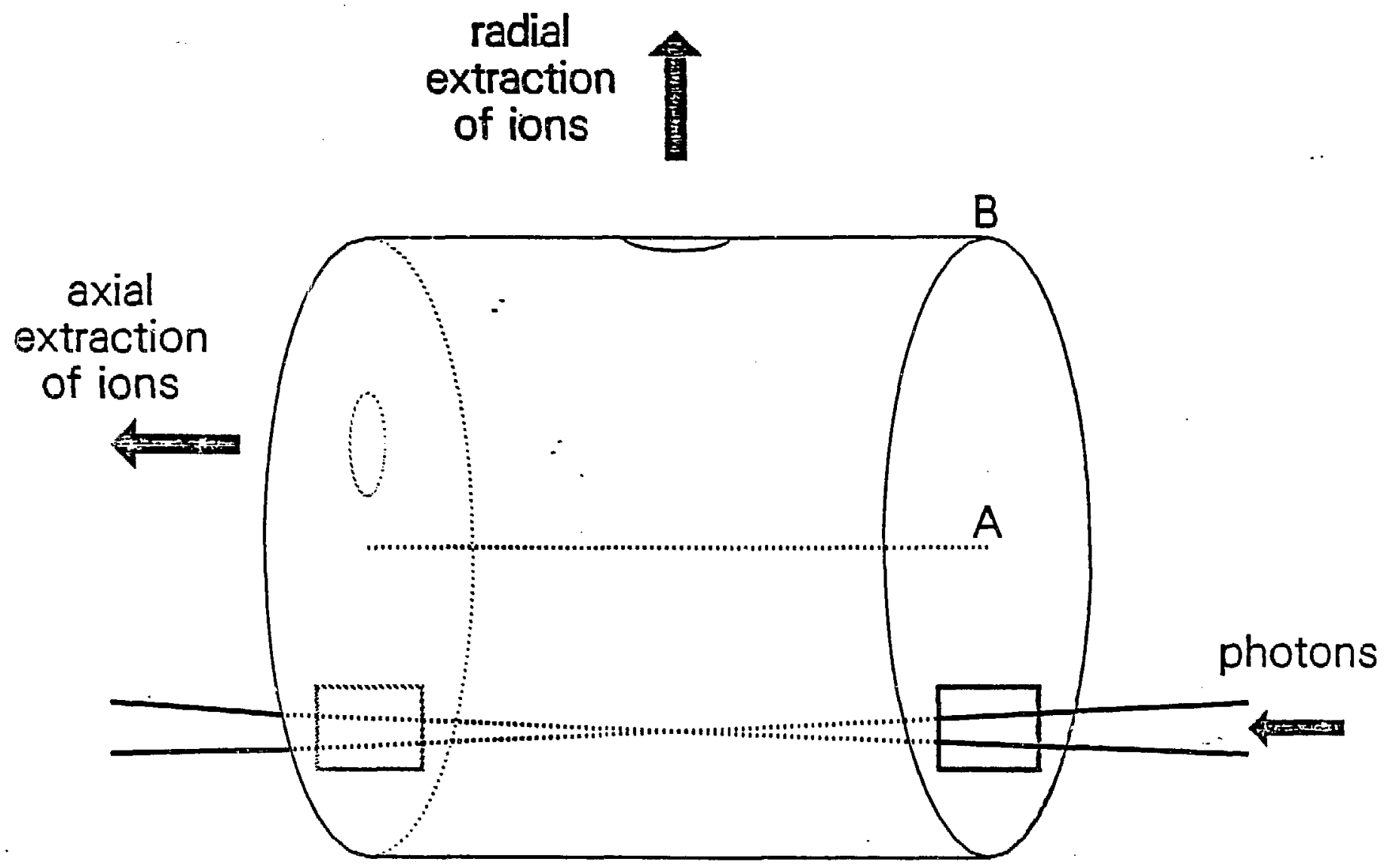


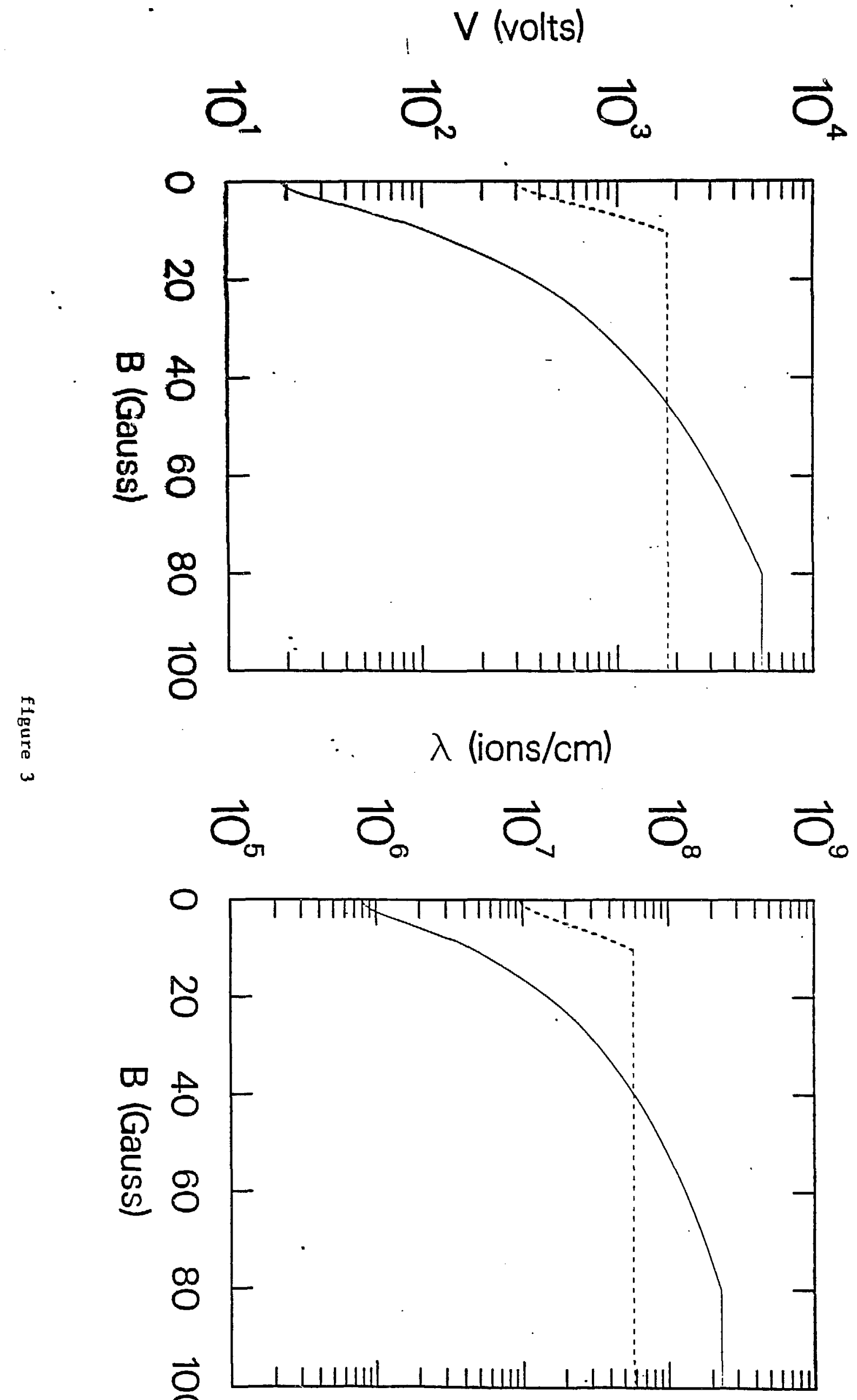




\section{PENNING ION TRAP}

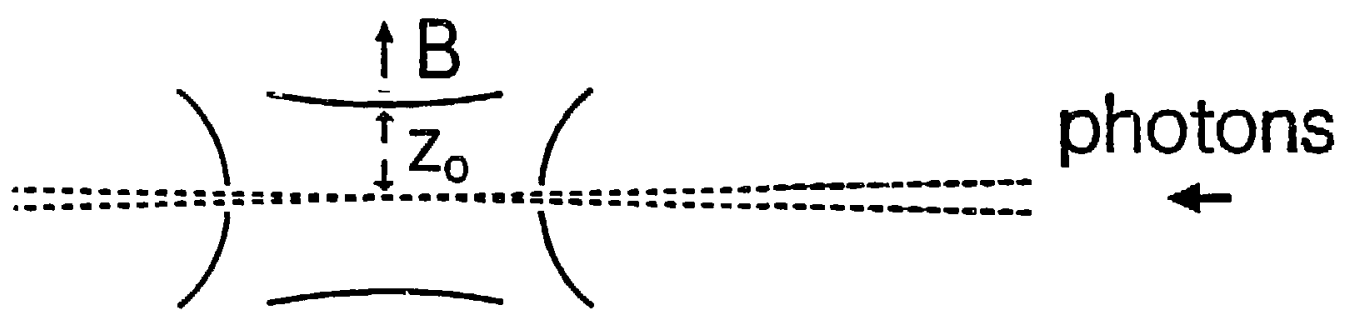

Trap ring electrodes

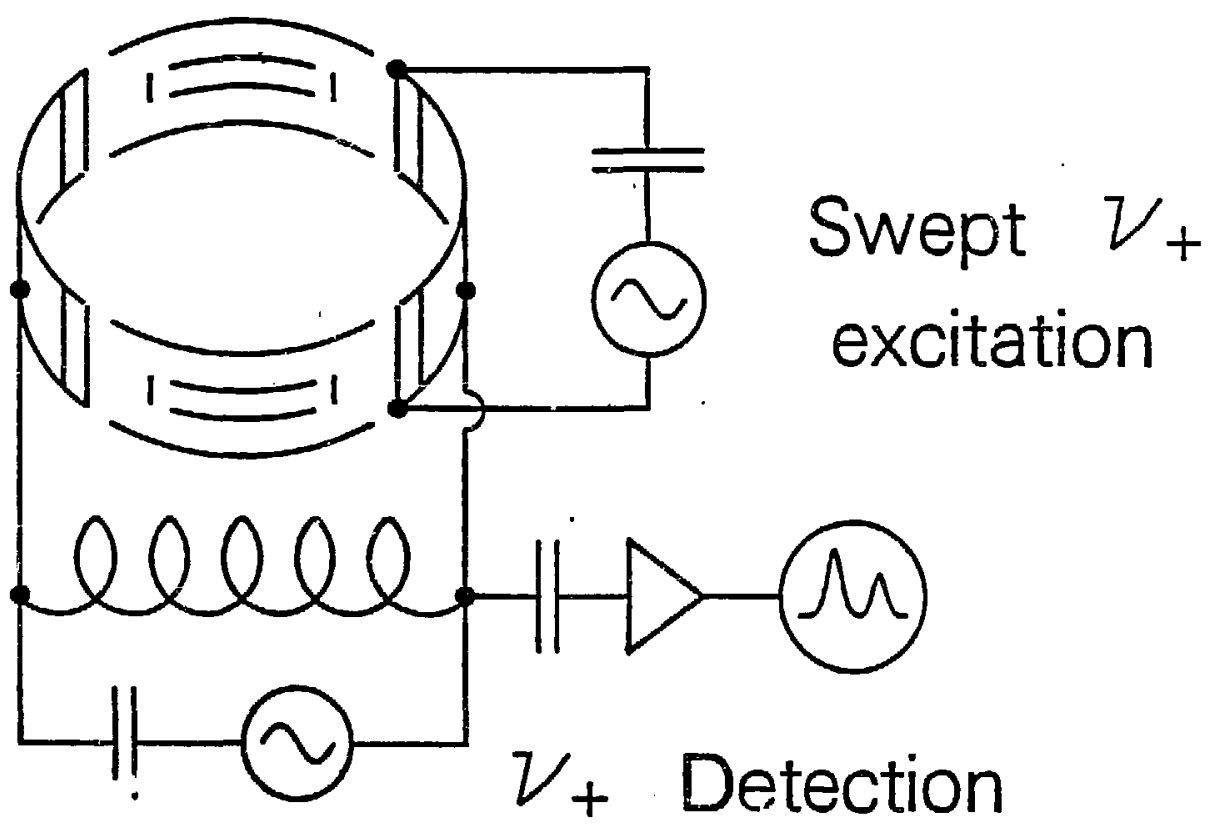

